\title{
[18F]DPA-714 PET Imaging for the Quantitative Evaluation of Early Spatiotemporal Changes of Neuroinflammation in Rat Brain Following Status Epilepticus
}

\section{Kenichi Kaneko}

RIKEN Center for Biosystems Dynamics Research

\section{Satsuki Irie}

RIKEN Center for Biosystems Dynamics Research

\section{Aya Mawatari}

RIKEN Center for Biosystems Dynamics Research

\section{Ami Igesaka}

RIKEN Center for Biosystems Dynamics Research

\section{Di Hu}

RIKEN Center for Biosystems Dynamics Research Library: Rigaku Kenkyujo Seimei Kino Kagaku Kenkyu Center Toshoshitsu

\section{Takayoshi Nakaoka}

RIKEN Center for Biosystems Dynamics Research

\section{Emi Hayashinaka}

RIKEN Center for Biosystems Dynamics Research

\section{Yasuhiro Wada}

RIKEN Center for Biosystems Dynamics Research

\section{Hisashi Doi}

RIKEN Center for Biosystems Dynamics Research

\section{Yasuyoshi Watanabe}

RIKEN Center for Biosystems Dynamics Research

\section{Yilong Cui ( $\nabla$ cuiyl@riken.jp )}

RIKEN Center for Biosystems Dynamics Research

https://orcid.org/0000-0002-8302-1899

\section{Research Article}

Keywords: [18F]DPA-714, anti-inflammatory therapies, epilepsy, neuroinflammation, microglia, spatiotemporal characteristics

Posted Date: January 17th, 2022 
DOl: https://doi.org/10.21203/rs.3.rs-892541/v2

License: (c) (1) This work is licensed under a Creative Commons Attribution 4.0 International License. Read Full License

Version of Record: A version of this preprint was published at European Journal of Nuclear Medicine and Molecular Imaging on February 14th, 2022. See the published version at https://doi.org/10.1007/s00259022-05719-7. 


\section{Abstract \\ Background}

Most antiepileptic drug therapies are symptomatic and adversely suppress normal brain function by nonspecific inhibition of neuronal activity. In recent times, growing evidence has suggested that neuroinflammation triggered by epileptic seizures might be involved in the pathogenesis of epilepsy. Although the potential effectiveness of anti-inflammatory treatment for curing epilepsy has been extensively discussed, the limited quantitative data regarding spatiotemporal characteristics of neuroinflammation after epileptic seizures makes it difficult to be realized. We quantitatively analyzed the spatiotemporal changes in neuroinflammation in the early phase after status epilepticus in rats, using translocator protein (TSPO) positron emission tomography (PET) imaging, which has been widely used for the quantitative evaluation of neuroinflammation in several animal models of CNS disease.

\section{Methods}

The second-generation TSPO PET probe, $\left[{ }^{18} \mathrm{~F}\right] \mathrm{DPA}-714$, was used for brain-wide quantitative analysis of neuroinflammation in the brains of rats, when the status epilepticus was induced by subcutaneous injection of kainic acid (KA, $15 \mathrm{mg} / \mathrm{kg}$ ) into those rats. A series of $\left[{ }^{18} \mathrm{~F}\right] \mathrm{DPA}-714$ PET scans were performed at 1, 3, 7, and 15 days after status epilepticus, and the corresponding histological changes, including activation of microglia and astrocytes, were confirmed by immunohistochemistry.

\section{Results}

Apparent accumulation of $\left[{ }^{18} \mathrm{~F}\right] \mathrm{DPA}-714$ was observed in several KA-induced epileptogenic regions, such as the amygdala, piriform cortex, ventral hippocampus, mediodorsal thalamus, and cortical regions 3 days after status epilepticus, and was reversibly displaced by unlabeled PK11195 (1 mg/kg). Consecutive $\left[{ }^{18} \mathrm{~F}\right] \mathrm{DPA}-714$ PET scans revealed that accumulation of $\left[{ }^{18} \mathrm{~F}\right] \mathrm{DPA}-714$ was focused in the KA-induced epileptogenic regions from 3 days after status epilepticus and was further maintained in the amygdala and piriform cortex until 7 days after status epilepticus. Immunohistochemical analysis revealed that activated microglia but not reactive astrocytes, were correlated with $\left[{ }^{18} \mathrm{~F}\right] \mathrm{DPA}-714$ accumulation in the KAinduced epileptogenic regions for at least 1 week after status epilepticus.

\section{Conclusions}

These results indicate that the early spatiotemporal characteristics of neuroinflammation quantitatively evaluated by $\left[{ }^{18} \mathrm{~F}\right] \mathrm{DPA}-714$ PET imaging provides valuable evidence for developing new antiinflammatory therapies for epilepsy. The predominant activation of microglia around epileptogenic regions in the early phase after status epilepticus could be a crucial therapeutic target for curing epilepsy. 


\section{Introduction}

Epilepsy is a chronic neurological disorder characterized by spontaneous recurrent seizures caused by neuronal hyperexcitability and hypersynchrony in the brain, and approximately 50 million people worldwide suffer from epilepsy [1,2]. Current antiepileptic drug therapy is effective in the majority of patients with epilepsy; however, most of the treatments are symptomatic and adversely affect normal brain function by nonspecific suppression of neuronal activity [3]. Moreover, approximately one-third of patients with epilepsy are pharmaco-resistant to the currently available antiepileptic drugs [4, 5], suggesting that the development of alternative pharmacological targets for curative therapy of epilepsy is strongly desired.

Accumulating evidence suggests that neuroinflammation induced by epileptic seizures is involved in the pathogenesis of epilepsy $[2,6-8]$. Clinical and preclinical studies have demonstrated that various proinflammatory mediators, such as IL-1 $\beta$ and TNF-a, are upregulated in the epileptic tissues $[9,10]$, and antiinflammatory manipulations effectively suppress epileptic seizures [11, 12]. Inflammation in the brain involves activation of glial cells, such as microglia and astrocytes, which have been reported to modulate neuronal excitability in epileptic foci $[2,9,13,14]$. The pro-inflammatory mediators released by the activated microglia have been reported to promote neuronal excitability and seizures in preclinical experiments [15]. Changes in the morphology and membrane protein composition in activated astrocytes in the epileptic foci cause functional impairment, buffering the ions, water, and neurotransmitters, which might lead to neuronal hyperexcitability and hypersynchrony [14]. These observations suggest that activated glial cells in the epileptic foci could be a curative therapeutic target for epilepsy. Although the potential effectiveness of anti-inflammatory strategies based on activated glial cells in the epileptic foci has been extensively discussed, quantitative evaluation of the spatiotemporal characteristics of glial activation following epileptic seizures is limited.

The spatiotemporal changes in neuroinflammation in the brain could be quantitatively assessed by noninvasive positron emission tomography (PET) imaging using radiolabeled molecular probes targeting specific biomarkers expressed in the activated glial cells. A mitochondrial outer membrane protein, $18 \mathrm{kD}$ translocator protein (TSPO), is generally recognized as a biomarker of activated microglia in the brain due to its unique expression property; it is expressed at low levels in the resting microglia but is markedly upregulated by activation in response to neuroinflammation $[16,17]$. Several chemical compounds have been designed and synthesized as specific PET probes for targeting the TSPO, including carbon-11 labeled PK11195 ( $\left.\left[{ }^{11} \mathrm{C}\right] \mathrm{PK} 11195\right)$, the first radiolabeled non-benzodiazepine-type PET probe for TSPO [18-22]. Due to the development of these PET probes, TSPO-PET imaging has been used extensively for the quantitative analysis of activated microglia in various diseases of the central nervous system in humans and animals, such as stroke, multiple sclerosis, Alzheimer's disease, and Parkinson's disease [23-29]. In line, attempts have also been made to reveal the neuroinflammation induced by epileptic seizures in animals and humans using $\left[{ }^{11} \mathrm{C}\right] \mathrm{PK} 11195$ or other second-generation TSPO ligands, including $\left[{ }^{18}\right.$ F]DPA-714 [30-33]. However, because of limited challenges and varied investigation designs, the detailed spatiotemporal dynamics of inflammatory glial cells in the pathophysiology of epilepsy are not 
fully understood. In this study, we quantitatively analyzed the early spatiotemporal changes in neuroinflammation following epileptic seizures using TSPO PET imaging with $\left[{ }^{18} \mathrm{~F}\right] \mathrm{DPA}-714$ which has improved signal-to-noise ratio and better imaging sensitivity than $\left[{ }^{11} \mathrm{C}\right] \mathrm{PK} 11195$. We found that specific accumulation of $\left[{ }^{18} \mathrm{~F}\right] \mathrm{DPA}-714$ was focused in the KA-induced epileptogenic regions from 3 days after epileptic seizures and activated microglia were predominantly responsible for the $\left[{ }^{18} \mathrm{~F}\right] \mathrm{DPA}-714$ accumulation in the KA-induced epileptogenic regions.

\section{Methods}

\section{Animals}

Thirty-three male Sprague-Dawley rats (7-10 weeks old) were obtained from Japan SLC (Hamamatsu, Shizuoka, Japan). The rats were housed in a light-and temperature-controlled room (approximately $22^{\circ} \mathrm{C}$, lights on at 8:00 and off at 20:00) and allowed free access to food and water. All experimental animal protocols were approved by the Animal Care and Use Committee of RIKEN, Kobe Branch, and were performed in accordance with the Principles of Laboratory Animal Care (National Institutes of Health Publication No. 85-23, revised 2011).

\section{Radiochemistry}

$\left[{ }^{18} \mathrm{~F}\right] \mathrm{DPA}-714$ was synthesized as reported previously by Kassiou's group [34]. The product was identified and purified by high-performance liquid chromatography on a COSMOSIL C18-AR-II column $(10 \times 250$ $\mathrm{mm}$, Nacalai, Kyoto, Japan). The molar activity ranged from 33 to $160 \mathrm{GBq} / \mu \mathrm{mol}$. The radiochemical purity analyzed by HPLC was greater than $99 \%$.

\section{Status epilepticus model}

To assess the early spatiotemporal changes in neuroinflammation after epileptic seizures, pharmacological status epilepticus was induced by systemic administration of kainic acid (KA), the most extensively used and well-validated animal model of human temporal lobe epilepsy [35]. Rats were subcutaneously injected with KA (15 mg/kg; Cayman Chemical, Ann Arbor, MI, USA) dissolved in saline at a concentration of $7.5 \mathrm{mg} / \mathrm{mL}$. Status epilepticus was observed for 4 hours and scored according to the Racine scale [36]. Rats showing class $\vee$ seizures (rearing with falling, tonic-clonic convulsions, bouncing, and continuous automatisms) were used in PET imaging and immunohistochemistry.

\section{PET Scans}

All PET scans were performed on a microPET Focus220 (Siemens, Knoxville, TN, USA), which was designed for small laboratory animals. Rats were anesthetized with $1.5 \%$ isoflurane and nitrous oxide/oxygen (7:3) and placed in the prone position in the PET scanner gantry. During the PET scan, the body temperature was maintained at $37^{\circ} \mathrm{C}$ using a small animal warmer connected to a thermometer (BWT-100A; Bio Research Center, Nagoya, Japan). A 60-min emission scan was performed immediately after a bolus injection of $\left[{ }^{18} \mathrm{~F}\right] \mathrm{DPA}-714(\approx 100 \mathrm{MBq}$ per animal) via a cannula inserted into the tail vein; 
the energy window was 400-650 keV with a coincidence time window of $6 \mathrm{~ns}$. Emission data were collected in the list mode and sorted into dynamic sinograms $(6 \times 10 \mathrm{~s}, 6 \times 30 \mathrm{~s}, 11 \times 60 \mathrm{~s}$, and $15 \times 180 \mathrm{~s}$, for

a total of 38 frames). The acquired data were reconstructed by standard 2D-filtered back projection (ramp filter, cutoff frequency at 0.5 cycles per pixel) after Fourier rebinning for quantification and by a statistical maximum a posteriori probability algorithm (MAP) (12 iterations with point spread function correction) for image registration.

In displacement experiments, $1 \mathrm{mg} / \mathrm{kg}$ unlabeled PK11195 (Abcam, Cambridge, UK) was injected at 20 min after the administration of $\left[{ }^{18} \mathrm{~F}\right] \mathrm{DPA}-714$.

\section{PET Image Analysis}

PET images were co-registered to an MRI template using the PMOD imaging processing software (version 3.6, PMOD Technologies, Zürich, Switzerland), and each region of interest (ROI) was defined according to the MRI template. PET images were expressed as standardized uptake values (SUVs). The decaycorrected mean value in each ROI was used to generate regional time-activity curves (TACs). For the quantitative analysis, the cerebellar white matter was defined as the reference region, and the binding potential (BP) was calculated using the simplified reference tissue model (SRTM) $[30,37,38]$ in the PMOD software package.

\section{Immunohistochemistry}

Rats were anesthetized with $5 \%$ isoflurane and perfused transcardially with $4 \%$ paraformaldehyde in 0.1 $\mathrm{M}$ phosphate-buffered saline ( $\mathrm{pH}$ 7.4). The brain was removed and post-fixed with the same fixative at $4^{\circ} \mathrm{C}$ overnight. Coronal brain sections ( $50 \mu \mathrm{m}$ thickness) were cut using a cryostat (CryoStar NX70, Thermo Fisher Scientific, Waltham, MA, USA). To examine the activation of glia, serial coronal brain sections were incubated with mouse anti-CD11b monoclonal antibody (1: 5000, MCA275R, Bio-Rad, Hercules, CA,USA) for microglia, or mouse anti-GFAP monoclonal antibody (1: 20000, G3893, SigmaAldrich, MO, USA) for astrocytes at $4^{\circ} \mathrm{C}$ for 18 hours. Immunoreactivity was visualized using the Vectastain ABC kit (Vector Laboratories, Burlingame, CA, USA) with 3, 3'-diaminobenzidine.

For the correlation analysis between the CD11b-positive activated microglia and $\left[{ }^{18} \mathrm{~F}\right] \mathrm{DPA}-714$ accumulation in the brain, semi-quantitative measurement of CD11b immunohistochemical staining was performed using ImageJ software ( $\mathrm{NIH}$, Bethesda, MD, USA) in 4 rats which have also examined [ $\left.{ }^{18} \mathrm{~F}\right] \mathrm{DPA}-$ 714 PET scan, as previously reported [39]. Eight square regions of interest (ROIs) were delineated on the CD11b-stained coronal brain section including the epileptogenic regions (Fig. 6C). In order to quantify $\mathrm{CD} 11 \mathrm{~b}$ immunostaining, the optical density for each ROI was calculated and normalized by an internal reference ROI (red square in Fig. $6 \mathrm{C}$ ) delineated on the same coronal brain section.

\section{Statistical Analysis}

All results are expressed as mean \pm SE. A two-way analysis of variance (ANOVA) with Bonferroni's multiple-comparison procedure was used to assess differences in the temporal changes in BP values 
between the ROls. A Pearson's test was used for correlation analysis between the CD11b immunostaining and corresponding $\left[{ }^{18} \mathrm{~F}\right] \mathrm{DPA}-714$ accumulation in the brain. Statistical significance was set at $P<0.05$.

\section{Results}

\section{Accumulation of [ $\left.{ }^{18} \mathrm{~F}\right] \mathrm{DPA}-714$ in the brain 3 days after status epilepticus}

To assess brain-wide changes in neuroinflammation in the early phase after epileptic seizures, we performed [ $\left.{ }^{18} \mathrm{~F}\right] \mathrm{DPA}-714$ PET scans in rats showing class $V$ seizures, 3 days after the subcutaneous injection of KA (15 mg/kg). As shown in Fig. 1, apparent accumulation of [ $\left.{ }^{18} \mathrm{~F}\right] \mathrm{DPA}-714$ was observed in several KA-induced epileptogenic regions: higher accumulation was observed in the limbic structures, such as the amygdala and piriform and entorhinal cortices, as well as in the ventral hippocampus. Moderate accumulation was seen in the widespread cerebral cortical regions and mediodorsal thalamus, while no obvious accumulation was observed in the cerebellum (Fig. 1). The brain pharmacokinetics of $\left[{ }^{18} \mathrm{~F}\right] \mathrm{DPA}-714$ in these KA-induced epileptogenic regions were similar (Fig. 2). The brain uptake of $\left[{ }^{18} \mathrm{~F}\right] \mathrm{DPA}-714$ reached a peak within the first minute after the bolus injection of radiotracer and decreased gradually thereafter in the amygdala, ventral hippocampus, and entorhinal cortex. Meanwhile, the brain uptake of $\left[{ }^{18} \mathrm{~F}\right] \mathrm{DPA}-714$ decreased rapidly in the cerebellum-white region after reaching the peak, further indicating a lack of $\left[{ }^{18} \mathrm{~F}\right] \mathrm{DPA}-714$ specific binding in this region.

Subsequently, the specific binding of $\left[{ }^{18} \mathrm{~F}\right] \mathrm{DPA}-714$ in these KA-induced epileptogenic regions was confirmed by in vivo displacement experiments with unlabeled PK11195, 3 days after status epilepticus. As shown in Fig. 3, accumulation of $\left[{ }^{18} \mathrm{~F}\right] \mathrm{DPA}-714$ in the epileptogenic region (amygdala) abruptly decreased to the level of the reference region (cerebellum) immediately after injection of $1 \mathrm{mg} / \mathrm{kg}$ unlabeled PK11195, at 20 min after the injection of $\left[{ }^{18} \mathrm{~F}\right] \mathrm{DPA}-714$, indicating that $\left[{ }^{18} \mathrm{~F}\right] \mathrm{DPA}-714$ was reversibly displaced by unlabeled PK11195.

\section{Spatiotemporal changes in [ $\left.{ }^{18} \mathrm{~F}\right] \mathrm{DPA}-714$ accumulation in the brain after status epilepticus}

We then examined the spatiotemporal changes in neuroinflammation following epileptic seizures through four consecutive PET scans with $\left[{ }^{18} \mathrm{~F}\right] \mathrm{DPA}-714$ on $1,3,7$, and 15 days after status epilepticus. One day after status epilepticus, the accumulation of $\left[{ }^{18} \mathrm{~F}\right] \mathrm{DPA}-714$ was barely seen in the brain, except for the cerebral ventricles and surrounding circumventricular area, similar to that in the normal rat brain (Fig. 4). As shown in Figs. 1 and 4, the apparent accumulation of $\left[{ }^{18} \mathrm{~F}\right] \mathrm{DPA}-714$ was observed in several KAinduced epileptogenic regions from 3 days after status epilepticus. The accumulation of $\left[{ }^{18} \mathrm{~F}\right] \mathrm{DPA}-714$ was more evident in the limbic regions, including the amygdala, piriform cortex, mediodorsal thalamus, and ventral hippocampus, at 7 days after status epilepticus. Quantitative analysis revealed that the BP in 
the ventral hippocampus and most KA-induced epileptogenic regions peaked at 3 days after status epilepticus, whereas BP in the amygdala and piriform cortex was maintained until 7 days after status epilepticus and decreased gradually thereafter (Fig. 5).

\section{Immunohistochemistry}

Finally, the activation of the microglia and astrocytes were confirmed by immunohistochemistry at 7 days after status epilepticus. As shown in Fig. 6A, abundant CD11b-positive activated microglia were observed in the KA-induced epileptogenic regions, such as the amygdala, piriform cortex, and hippocampus. In contrast, GFAP-positive activated astrocytes were broadly observed throughout the brain. Notably, activated astrocytes were rarely observed in the central area of the amygdala and dorsal thalamus, in which the high level of $\left[{ }^{18} \mathrm{~F}\right] \mathrm{DPA}-714$ accumulation was observed (Fig. 6B). These results suggest that activated microglia could be the main source of $\left[{ }^{18} \mathrm{~F}\right] \mathrm{DPA}-714$ accumulation in the KA-induced epileptogenic regions at least for 1 week after status epilepticus.

To further confirm whether the localized microglia activation in the epileptogenic regions is correlated with the accumulation of $\left[{ }^{18} \mathrm{~F}\right] \mathrm{DPA}-714$ at 7 days after status epilepticus, we performed a semiquantitative measurement of CD11b immunostaining and compared that with the corresponding accumulation of $\left[{ }^{18} \mathrm{~F}\right] \mathrm{DPA}-714$ in the same rats. As shown in Fig. $6 \mathrm{C}$, the intensity of CD11b immunostaining was significantly correlated with the accumulation of [18F]DPA-714 in these rats at 7 days after status epilepticus.

\section{Discussion}

In this study, we performed brain-wide quantitative evaluation of the early spatiotemporal changes in neuroinflammation induced by status epilepticus using [ ${ }^{18}$ F]DPA-714 PET imaging and found that neuroinflammation was predominantly restricted to the KA-induced epileptogenic regions in the early phase after epileptic seizures. Moreover, activated microglia were predominantly responsible for the specific accumulation of $\left[{ }^{18} \mathrm{~F}\right] \mathrm{DPA}-714$ in these KA-induced epileptogenic regions during the critical therapeutic time window for epileptic seizures. The results of the study show that: 1) specific accumulation of $\left[{ }^{18} \mathrm{~F}\right] \mathrm{DPA}-714$ was observed in the KA-induced epileptogenic regions at 3 days after status epilepticus induced by systemic injection of $\mathrm{KA} ; 2)$ a series of $\left[{ }^{18} \mathrm{~F}\right] \mathrm{DPA}-714$ PET imaging studies revealed that neuroinflammation initially appeared in the ventral hippocampus and surrounding cortices at 3 days after status epilepticus, and was further sustained in the nearby amygdala and piriform cortex until 7 days after status epilepticus; 3 ) immunohistochemical analyses revealed that the activated microglia but not reactive astrocytes were correlated with the $\left[{ }^{18} \mathrm{~F}\right] \mathrm{DPA}-714$ accumulation in the KAinduced epileptogenic regions at least for 1 week after status epilepticus.

Over 50 chemical compounds have been designed and synthesized as TSPO PET probes for the quantitative assessment of inflammatory processes in the brain [18-21, 24, 26, 27, 29, 40]. [ $\left.{ }^{11} \mathrm{C}\right] \mathrm{PK} 11195$, 
the first radiolabeled non-benzodiazepine-type PET probe for TSPO has been extensively used as the gold standard for TSPO imaging in preclinical and clinical studies [24, 28, 29, 40]. Nevertheless, several disadvantages have restricted its practical application in the quantitative estimation of neuroinflammation, such as poor signal-to-noise ratio, low brain uptake, and high levels of nonspecific binding [41]. The pyrazolopyrimidine compound [ $\left.{ }^{18} \mathrm{~F}\right] \mathrm{DPA}-714$, which has higher affinity and better bloodbrain barrier permeability, was developed by Kassiou's group and has been used for the quantitative assessment of neuroinflammation in CNS diseases, including stroke [26], trauma [42], Alzheimer's disease [43], and amyotrophic lateral sclerosis [44]. Using an acute focal neuroinflammation model, Chauveau et al. [38] demonstrated that $\left[{ }^{18}\right.$ F]DPA-714 has an increased accumulation in the inflamed area and a reduced nonspecific binding in normal areas compared to $\left[{ }^{11} \mathrm{C}\right] \mathrm{PK} 11195$. Similarly, we also revealed that the specific binding of $\left[{ }^{18} \mathrm{~F}\right] \mathrm{DPA}-714$ in the KA-induced epileptogenic regions was significantly higher than that of $\left[{ }^{11} \mathrm{C}\right] \mathrm{PK} 11195$ in the status epilepticus model rats using the same animals on the same day (unpublished data). With the improved imaging capability of $\left[{ }^{18} \mathrm{~F}\right] \mathrm{DPA}-714$, we demonstrated that the accumulation of $\left[{ }^{18} \mathrm{~F}\right] \mathrm{DPA}-714$ was significantly increased in the KA-induced epileptogenic regions at 3 days after status epilepticus (Figs. 1 and 4). Furthermore, the accumulation of $\left[{ }^{18} \mathrm{~F}\right] \mathrm{DPA}-714$ in the KA-induced epileptogenic regions was successfully displaced by unlabeled PK11195 (Fig. 3), suggesting that $\left[{ }^{18} \mathrm{~F}\right] \mathrm{DPA}-714$ was specifically bound to TSPO even in the early phase after epileptic seizures. Considering together, these observations suggest that PET imaging with [ $\left.{ }^{18} \mathrm{~F}\right] \mathrm{DPA}-714$, the second-generation TSPO radioligand, is a reliable and higher sensitive imaging tool for the quantitative evaluation of changes in neuroinflammatory processes throughout the brain in the early phase of epileptic seizures.

In the present study, we successfully evaluated the early spatiotemporal changes in neuroinflammation following status epilepticus induced by systemic injection of KA, using $\left[{ }^{18} \mathrm{~F}\right] \mathrm{DPA}-714$ PET imaging. Elucidation of the spatiotemporal changes in neuroinflammation during different stages of seizure, especially the early phase after epileptic seizures, could be crucial for understanding the pathophysiological mechanisms of epileptogenesis and improving the treatment methods. Several TSPO PET imaging studies have attempted to reveal spatiotemporal changes in neuroinflammation following epileptic seizures [30-32]. Brackhan et al. [30] reported that accumulation of [ $\left.{ }^{11} \mathrm{C}\right] \mathrm{PK} 11195$ was observed in most brain areas until 5-7 days after status epilepticus (rats showing stage 4 or 5 seizures according to the Racine scale) induced by systemic administration of pilocarpine-lithium, and was subsequently more concentrated in epileptogenic regions thereafter. Similarly, we demonstrated that significant accumulation of $\left[{ }^{18} \mathrm{~F}\right] \mathrm{DPA}-714$ was restricted to the KA-induced epileptogenic regions from 3 days after systemic KA-injection induced status epilepticus (Figs. 1 and 4). Moreover, we also clearly demonstrated the spatiotemporal difference in $\left[{ }^{18} \mathrm{~F}\right] \mathrm{DPA}-714$ accumulation between the KA-induced epileptogenic regions, such as the BP for $\left[{ }^{18} \mathrm{~F}\right] \mathrm{DPA}-714$ in most KA-induced epileptogenic regions peaked at 3 days after status epilepticus but remained until 7 days after status epilepticus in the amygdala and piriform cortex (Fig. 5). These observations suggest that $\left[{ }^{18} \mathrm{~F}\right] \mathrm{DPA}-714 \mathrm{PET}$ imaging enables to evaluate has greater 
sensitivity in detecting the early phase of neuroinflammation following epileptic seizures than $\left[{ }^{11}\right.$ C $]$ PK11195.

In the present study, we also demonstrated that activated microglia were predominantly responsible for the specific accumulation of $\left[{ }^{18} \mathrm{~F}\right] \mathrm{DPA}-714$ in the KA-induced epileptogenic regions, at least within the first week. Although TSPO was initially recognized as a specific biomarker for activated microglia $[16,17]$, some results have also demonstrated that TSPO might also be upregulated in reactive astrocytes $[27,45]$. Using CNS neurodegenerative disease animal models, Ji et al.[27] clearly demonstrated that the dominant expression of TSPO (originally called PBR, peripheral benzodiazepine receptor) in the activated astrocytes was associated with minimal or reversible neuronal injury, whereas dominant TSPO expression in the activated microglia was associated with irreversible neuronal insults. Whereas, TSPO expression during epileptogenesis and chronic phase of epileptic seizures correlated well with microglial activation rather than with reactive astrocytes $[30,46]$. Consistent with these observations, we found that CD11b-positive activated microglia, but not GFAP-positive reactive astrocytes, were concentrated in the KA-induced epileptogenic regions, which showed a high accumulation of $\left[{ }^{18} \mathrm{~F}\right] \mathrm{DPA}-714$ (Fig. 6). Activation of microglia and astrocytes has been implicated as a crucial process in the pathophysiology of epileptogenesis $[2,9,14]$. Activated microglia might contribute to neuronal hyperexcitability and susceptibility to seizures through the release of inflammatory mediators, such as IL-1 $\beta$ and TNF- $\alpha[2,47]$. Pharmacological interventions that inhibit microglial activation or block IL-1 signaling can suppress epileptic seizures $[11,12]$. Reactive astrocytes induced by status epilepticus have been reported to lose homeostatic buffering capabilities, which might facilitate spontaneous seizures [48, 49]. Recent studies demonstrated that microglia are activated immediately after status epilepticus $[47,50]$ and might contribute to astrocyte reactivation [51]. In line with this finding, Sano et al.[52] demonstrated that early inhibition of microglial activation after the early phase of status epilepticus suppresses astrocyte reactivation and epileptic seizures. These observations suggest that microglial activation in the early period of epileptic seizures could be a crucial therapeutic target for pharmacological intervention, and $\left[{ }^{18}\right.$ F]DPA-714 PET imaging allows quantitative evaluation of spatiotemporal changes in microglial activation in the early phase after epileptic seizures.

Growing evidence has demonstrated that the functional role of activated microglia in CNS diseases is dependent on the varied activation states, such as the classical M1 or alternative M2 phenotypes. The M1 and M2 phenotypes of activated microglia have been shown to dynamically change with the progression of CNS diseases, such as stroke [53], multiple sclerosis [54], and Alzheimer's disease [55], and to be involved in different pathophysiological processes of these diseases, respectively. Although the M1 and M2 phenotypes of activated microglia have also been observed in temporal lobe epilepsy model animal, the functional role of M1 and M2 microglia in epileptogenesis remains unclear [47, 56]. Since TSPO is a general biomarker for activated microglia expressed in both $\mathrm{M} 1$ and $\mathrm{M} 2$ phenotypes [56], a phenotypespecific PET ligand for activated microglia needs to be developed in the future. 
In conclusion, we demonstrated that $\left[{ }^{18} \mathrm{~F}\right] \mathrm{DPA}-714$ PET imaging is a reliable and sensitive tool for the quantitative evaluation of early spatiotemporal dynamics of neuroinflammation following status epilepticus in vivo. The brain-wide $\left[{ }^{18} \mathrm{~F}\right] \mathrm{DPA}-714 \mathrm{PET}$ imaging analysis showed that neuroinflammation focused in the epileptogenic regions and activated microglia were predominantly correlated with $\left[{ }^{18} \mathrm{~F}\right] \mathrm{DPA}-714$ accumulation at least for 1 week after status epilepticus. Given that most of the antiepileptic drug therapies are symptomatic and adversely affect normal brain function by suppressing neuronal activity nonspecifically, our results suggest that anti-inflammatory treatment based on microglial activation could be a curative therapeutic strategy for epileptic seizures and that $\left[{ }^{18} \mathrm{~F}\right] \mathrm{DPA}-714$ PET imaging could be a fascinating important tool for determining therapeutic windows and monitoring the therapeutic efficacy.

\section{Abbreviations}

CNS, central nervous system; $\left[{ }^{11} \mathrm{C}\right] \mathrm{PK} 11195,1-\left(2\right.$-chlorophenyl)- $N-\left[{ }^{11} \mathrm{C}\right]$ methyl- $N$-(1-methylpropyl)-3isoquinolinecarboxamide; $\left[{ }^{18} \mathrm{~F}\right] \mathrm{DPA}-714, \mathrm{~N}, \mathrm{~N}$-diethyl-2-[4-(2-[18 $\left.\mathrm{F}\right]$ fluoroethoxy)phenyl]-5,7dimethylpyrazolo[1,5-a]pyrimidine-3-acetamide; GFAP, glial fibrillary acidic protein; IL-1 $\beta$, interleukin 1beta; $\mathrm{KA}$, kainic acid; MRI, Magnetic Resonance Imaging; PET, positron emission tomography; ROI, region of interest; SRTM, simplified reference tissue model; SUV, standardized uptake value; TAC, time-activity curve; TNF-alpha: tumor necrosis factor-alpha; TSPO, translocator protein.

\section{Declarations}

\section{- Ethics approval and consent to participate}

All experimental animal protocols were approved by the Animal Care and Use Committee of RIKEN, Kobe Branch, and were performed in accordance with the Principles of Laboratory Animal Care (National Institutes of Health Publication No. 85-23, revised 2011).

\section{- Consent for publication}

Not applicable

\section{- Availability of data and materials}

The datasets used and/or analyzed during the current study are available from the corresponding author on reasonable request.

\section{- Competing interests}

The authors declare that they have no competing interests.

\section{- Funding}


This work was supported in part by AMED under Grant Numbers JP18dk0310068 to Y.W., JP20ak0101059 to Y.W. and Y.C., and JP20ak0101063 to Y.W. and Y.C.

\section{- Authors' contributions}

Ken-ichi Kaneko: experimental design, writing and revising the manuscript

Satsuki Irie: PET imaging analysis, immunohistochemical study and interpretation

Aya Mawatari: synthetic design and PET probe labeling

Ami Igesaka: synthetic design and PET probe labeling

Di Hu: immunohistochemical study and interpretation

Takayoshi Nakaoka: PET imaging analysis and interpretation

Emi Hayashinaka: PET imaging reconstruction and interpretation

Yasuhiro Wada: PET imaging reconstruction and interpretation

Hisashi Doi: PET probe labeling and interpretation, revising the manuscript

Yasuyoshi Watanabe: conception and design of study, and revising the manuscript

Yilong Cui: conception and design of study, interpreting data and revising the manuscript

\section{- Acknowledgements}

We are grateful to Masahiro Kurahashi of Sumitomo Heavy Industry Accelerator Service Ltd. for operation of the cyclotron. We appreciate Kayo Onoe of the RIKEN Center for Biosystems Dynamics Research for her expert technical assistance. This work was supported in part by AMED under Grant Numbers JP18dk0310068 to Y.W., JP20ak0101059 to Y.W. and Y.C., and JP20ak0101063 to Y.W. and Y.C.

\section{References}

1. Hauser WA, Rich SS, Lee JR, Annegers JF, Anderson VE: Risk of recurrent seizures after two unprovoked seizures.N Engl J Med 1998, 338:429-434.

2. Vezzani A, French J, Bartfai T, Baram TZ: The role of inflammation in epilepsy.Nat Rev Neuro/ 2011, 7:31-40.

3. Rogawski MA, Loscher W: The neurobiology of antiepileptic drugs.Nat Rev Neurosci 2004, 5:553-564.

4. Perucca E, French J, Bialer M: Development of new antiepileptic drugs: challenges, incentives, and recent advances.Lancet Neurol 2007, 6:793-804. 
5. Wiebe S, Jette N: Epilepsy surgery utilization: who, when, where, and why? Curr Opin Neurol 2012, 25:187-193.

6. Vitaliti G, Pavone P, Mahmood F, Nunnari G, Falsaperla R: Targeting inflammation as a therapeutic strategy for drug-resistant epilepsies: an update of new immune-modulating approaches. Hum Vaccin Immunother 2014, 10:868-875.

7. Vezzani A, Balosso S, Ravizza T: Neuroinflammatory pathways as treatment targets and biomarkers in epilepsy.Nat Rev Neurol 2019, 15:459-472.

8. Rana A, Musto AE: The role of inflammation in the development of epilepsy.J Neuroinflammation 2018, 15:144.

9. Amhaoul H, Staelens S, Dedeurwaerdere S: Imaging brain inflammation in epilepsy.Neuroscience 2014, 279:238-252.

10. Vezzani A, Granata T: Brain inflammation in epilepsy: experimental and clinical evidence.Epilepsia 2005, 46:1724-1743.

11. Abraham J, Fox PD, Condello C, Bartolini A, Koh S: Minocycline attenuates microglia activation and blocks the long-term epileptogenic effects of early-life seizures.Neurobiol Dis 2012, 46:425-430.

12. Maroso M, Balosso S, Ravizza T, lori V, Wright $\mathrm{Cl}$, French J, Vezzani A: Interleukin-1 beta biosynthesis inhibition reduces acute seizures and drug resistant chronic epileptic activity in mice.Neurotherapeutics 2011, 8:304-315.

13. Fujita S, Mizoguchi N, Aoki R, Cui Y, Koshikawa N, Kobayashi M: Cytoarchitecture-Dependent Decrease in Propagation Velocity of Cortical Spreading Depression in the Rat Insular Cortex Revealed by Optical Imaging. Cereb Cortex 2016, 26:1580-1589.

14. Devinsky O, Vezzani A, Najjar S, De Lanerolle NC, Rogawski MA: Glia and epilepsy: excitability and inflammation. Trends Neurosci 2013, 36:174-184.

15. Rodgers KM, Hutchinson MR, Northcutt A, Maier SF, Watkins LR, Barth DS: The cortical innate immune response increases local neuronal excitability leading to seizures.Brain 2009, 132:24782486.

16. Banati RB: Visualising microglial activation in vivo.Glia 2002, 40:206-217.

17. Chen MK, Guilarte TR: Translocator protein 18 kDa (TSPO): molecular sensor of brain injury and repair.Pharmacol Ther 2008, 118:1-17.

18. Wilms H, Claasen J, Rohl C, Sievers J, Deuschl G, Lucius R: Involvement of benzodiazepine receptors in neuroinflammatory and neurodegenerative diseases: evidence from activated microglial cells in vitro.Neurobiol Dis 2003, 14:417-424.

19. Owen DR, Gunn RN, Rabiner EA, Bennacef I, Fujita M, KreisI WC, Innis RB, Pike VW, Reynolds R, Matthews PM, Parker CA: Mixed-affinity binding in humans with 18-kDa translocator protein ligands.J Nucl Med 2011, 52:24-32.

20. Maeda J, Suhara T, Zhang MR, Okauchi T, Yasuno F, Ikoma Y, Inaji M, Nagai Y, Takano A, Obayashi S, Suzuki K: Novel peripheral benzodiazepine receptor ligand [11C]DAA1106 for PET: an imaging tool 
for glial cells in the brain.Synapse 2004, 52:283-291.

21. Le Fur G, Guilloux F, Rufat P, Benavides J, Uzan A, Renault C, Dubroeucq MC, Gueremy C: Peripheral benzodiazepine binding sites: effect of PK 11195, 1-(2-chlorophenyl)-N-methyl-(1-methylpropyl)-3 isoquinolinecarboxamide. II. In vivo studies.Life Sci 1983, 32:1849-1856.

22. Kim K, Kim H, Bae SH, Lee SY, Kim YH, Na J, Lee CH, Lee MS, Ko GB, Kim KY, et al: [(18)F]CB251 PET/MR imaging probe targeting translocator protein (TSPO) independent of its Polymorphism in a Neuroinflammation Model. Theranostics 2020, 10:9315-9331.

23. Zurcher NR, Loggia ML, Mullett JE, Tseng C, Bhanot A, Richey L, Hightower BG, Wu C, Parmar AJ, Butterfield RI, et al: [(11)C]PBR28 MR-PET imaging reveals lower regional brain expression of translocator protein (TSPO) in young adult males with autism spectrum disorder.Mol Psychiatry 2021, 26:1659-1669.

24. Price CJ, Wang D, Menon DK, Guadagno JV, Cleij M, Fryer T, Aigbirhio F, Baron JC, Warburton EA: Intrinsic activated microglia map to the peri-infarct zone in the subacute phase of ischemic stroke.Stroke 2006, 37:1749-1753.

25. Nutma E, Stephenson JA, Gorter RP, de Bruin J, Boucherie DM, Donat CK, Breur M, van der Valk P, Matthews PM, Owen DR, Amor S: A quantitative neuropathological assessment of translocator protein expression in multiple sclerosis.Brain 2019, 142:3440-3455.

26. Martin A, Boisgard R, Theze B, Van Camp N, Kuhnast B, Damont A, Kassiou M, Dolle F, Tavitian B: Evaluation of the PBR/TSPO radioligand [(18)F]DPA-714 in a rat model of focal cerebral ischemia.J Cereb Blood Flow Metab 2010, 30:230-241.

27. Ji B, Maeda J, Sawada M, Ono M, Okauchi T, Inaji M, Zhang MR, Suzuki K, Ando K, Staufenbiel M, et al: Imaging of peripheral benzodiazepine receptor expression as biomarkers of detrimental versus beneficial glial responses in mouse models of Alzheimer's and other CNS pathologies.J Neurosci 2008, 28:12255-12267.

28. Gerhard A, Pavese N, Hotton G, Turkheimer F, Es M, Hammers A, Eggert K, Oertel W, Banati RB, Brooks DJ: In vivo imaging of microglial activation with [11C](R)-PK11195 PET in idiopathic Parkinson's disease.Neurobiol Dis 2006, 21:404-412.

29. Cui Y, Takashima T, Takashima-Hirano M, Wada Y, Shukuri M, Tamura Y, Doi H, Onoe H, Kataoka Y, Watanabe Y: 11C-PK11195 PET for the in vivo evaluation of neuroinflammation in the rat brain after cortical spreading depression.J Nucl Med 2009, 50:1904-1911.

30. Brackhan M, Bascunana P, Postema JM, Ross TL, Bengel FM, Bankstahl M, Bankstahl JP: Serial Quantitative TSPO-Targeted PET Reveals Peak Microglial Activation up to 2 Weeks After an Epileptogenic Brain Insult.J Nucl Med 2016, 57:1302-1308.

31. Brackhan M, Bascunana P, Ross TL, Bengel FM, Bankstahl JP, Bankstahl M: [(18) F]GE180 positron emission tomographic imaging indicates a potential double-hit insult in the intrahippocampal kainate mouse model of temporal lobe epilepsy.Epilepsia 2018, 59:617-626.

32. Nguyen DL, Wimberley C, Truillet C, Jego B, Caille F, Pottier G, Boisgard R, Buvat I, Bouilleret V: Longitudinal positron emission tomography imaging of glial cell activation in a mouse model of 
mesial temporal lobe epilepsy: Toward identification of optimal treatment windows.Epilepsia 2018, 59:1234-1244.

33. Bertoglio D, Amhaoul H, Goossens J, Ali I, Jonckers E, Bijnens T, Siano M, Wyffels L, Verhaeghe J, Van der Linden A, et al: TSPO PET upregulation predicts epileptic phenotype at disease onset independently from chronic TSPO expression in a rat model of temporal lobe epilepsy.Neuroimage Clin 2021, 31:102701.

34. James ML, Fulton RR, Vercoullie J, Henderson DJ, Garreau L, Chalon S, Dolle F, Costa B, Guilloteau D, Kassiou M: DPA-714, a new translocator protein-specific ligand: synthesis, radiofluorination, and pharmacologic characterization.J Nucl Med 2008, 49:814-822.

35. Sperk G, Lassmann H, Baran H, Seitelberger F, Hornykiewicz O: Kainic acid-induced seizures: doserelationship of behavioural, neurochemical and histopathological changes.Brain Res 1985, 338:289295.

36. Racine RJ: Modification of seizure activity by electrical stimulation. II. Motor seizure.Electroencephalogr Clin Neurophysiol 1972, 32:281-294.

37. Lammertsma AA, Hume SP: Simplified reference tissue model for PET receptor studies.Neuroimage 1996, 4:153-158.

38. Chauveau F, Van Camp N, Dolle F, Kuhnast B, Hinnen F, Damont A, Boutin H, James M, Kassiou M, Tavitian B: Comparative evaluation of the translocator protein radioligands 11C-DPA-713, 18F-DPA714, and 11C-PK11195 in a rat model of acute neuroinflammation.J Nucl Med 2009, 50:468-476.

39. Crowe AR, Yue W: Semi-quantitative Determination of Protein Expression using Immunohistochemistry Staining and Analysis: An Integrated Protocol.Bio Protoc 2019, 9:e3465.

40. Banati RB, Newcombe J, Gunn RN, Cagnin A, Turkheimer F, Heppner F, Price G, Wegner F, Giovannoni $\mathrm{G}$, Miller $\mathrm{DH}$, et al: The peripheral benzodiazepine binding site in the brain in multiple sclerosis: quantitative in vivo imaging of microglia as a measure of disease activity.Brain 2000, 123 ( Pt 11):2321-2337.

41. Chauveau F, Boutin H, Van Camp N, Dolle F, Tavitian B: Nuclear imaging of neuroinflammation: a comprehensive review of [11C]PK11195 challengers.Eur J Nucl Med Mol Imaging 2008, 35:23042319.

42. Wang Y, Yue X, Kiesewetter DO, Niu G, Teng G, Chen X: PET imaging of neuroinflammation in a rat traumatic brain injury model with radiolabeled TSPO ligand DPA-714. Eur J Nucl Med Mol Imaging 2014, 41:1440-1449.

43. Keller T, Lopez-Picon FR, Krzyczmonik A, Forsback S, Kirjavainen AK, Takkinen JS, Alzghool O, Rajander J, Teperi S, Cacheux F, et al: [(18)F]F-DPA for the detection of activated microglia in a mouse model of Alzheimer's disease.Nucl Med Biol 2018, 67:1-9.

44. Corcia P, Tauber C, Vercoullie J, Arlicot N, Prunier C, Praline J, Nicolas G, Venel Y, Hommet C, Baulieu $\mathrm{JL}$, et al: Molecular imaging of microglial activation in amyotrophic lateral sclerosis.PLoS One 2012, 7:e52941. 
45. Itzhak Y, Baker L, Norenberg MD: Characterization of the peripheral-type benzodiazepine receptors in cultured astrocytes: evidence for multiplicity. Glia 1993, 9:211-218.

46. Amhaoul H, Hamaide J, Bertoglio D, Reichel SN, Verhaeghe J, Geerts E, Van Dam D, De Deyn PP, Kumar-Singh S, Katsifis A, et al: Brain inflammation in a chronic epilepsy model: Evolving pattern of the translocator protein during epileptogenesis.Neurobiol Dis 2015, 82:526-539.

47. Benson MJ, Manzanero S, Borges K: Complex alterations in microglial M1/M2 markers during the development of epilepsy in two mouse models.Epilepsia 2015, 56:895-905.

48. Haj-Yasein NN, Jensen V, Vindedal GF, Gundersen GA, Klungland A, Ottersen OP, Hvalby O, Nagelhus EA: Evidence that compromised $K+$ spatial buffering contributes to the epileptogenic effect of mutations in the human Kir4.1 gene (KCNJ10). Glia 2011, 59:1635-1642.

49. Robel S, Buckingham SC, Boni JL, Campbell SL, Danbolt NC, Riedemann T, Sutor B, Sontheimer H: Reactive astrogliosis causes the development of spontaneous seizures.J Neurosci 2015, 35:33303345.

50. Vezzani A, Conti M, De Luigi A, Ravizza T, Moneta D, Marchesi F, De Simoni MG: Interleukin-1beta immunoreactivity and microglia are enhanced in the rat hippocampus by focal kainate application: functional evidence for enhancement of electrographic seizures.J Neurosci 1999, 19:5054-5065.

51. Liddelow SA, Guttenplan KA, Clarke LE, Bennett FC, Bohlen CJ, Schirmer L, Bennett ML, Munch AE, Chung WS, Peterson TC, et al: Neurotoxic reactive astrocytes are induced by activated microglia.Nature 2017, 541:481-487.

52. Sano F, Shigetomi E, Shinozaki Y, Tsuzukiyama H, Saito K, Mikoshiba K, Horiuchi H, Cheung DL, Nabekura J, Sugita K, et al: Reactive astrocyte-driven epileptogenesis is induced by microglia initially activated following status epilepticus.JCI Insight 2021, 6:e135391.

53. Hu X, Li P, Guo Y, Wang H, Leak RK, Chen S, Gao Y, Chen J: Microglia/macrophage polarization dynamics reveal novel mechanism of injury expansion after focal cerebral ischemia.Stroke 2012, 43:3063-3070.

54. Peferoen LA, Vogel DY, Ummenthum K, Breur M, Heijnen PD, Gerritsen WH, Peferoen-Baert RM, van der Valk P, Dijkstra CD, Amor S: Activation status of human microglia is dependent on lesion formation stage and remyelination in multiple sclerosis.J Neuropathol Exp Neurol 2015, 74:48-63.

55. Tang Y, Le W: Differential Roles of M1 and M2 Microglia in Neurodegenerative Diseases.Mol Neurobio/ 2016, 53:1181-1194.

56. Ali I, Chugh D, Ekdahl CT: Role of fractalkine-CX3CR1 pathway in seizure-induced microglial activation, neurodegeneration, and neuroblast production in the adult rat brain.Neurobiol Dis 2015, 74:194-203.

\section{Figures}




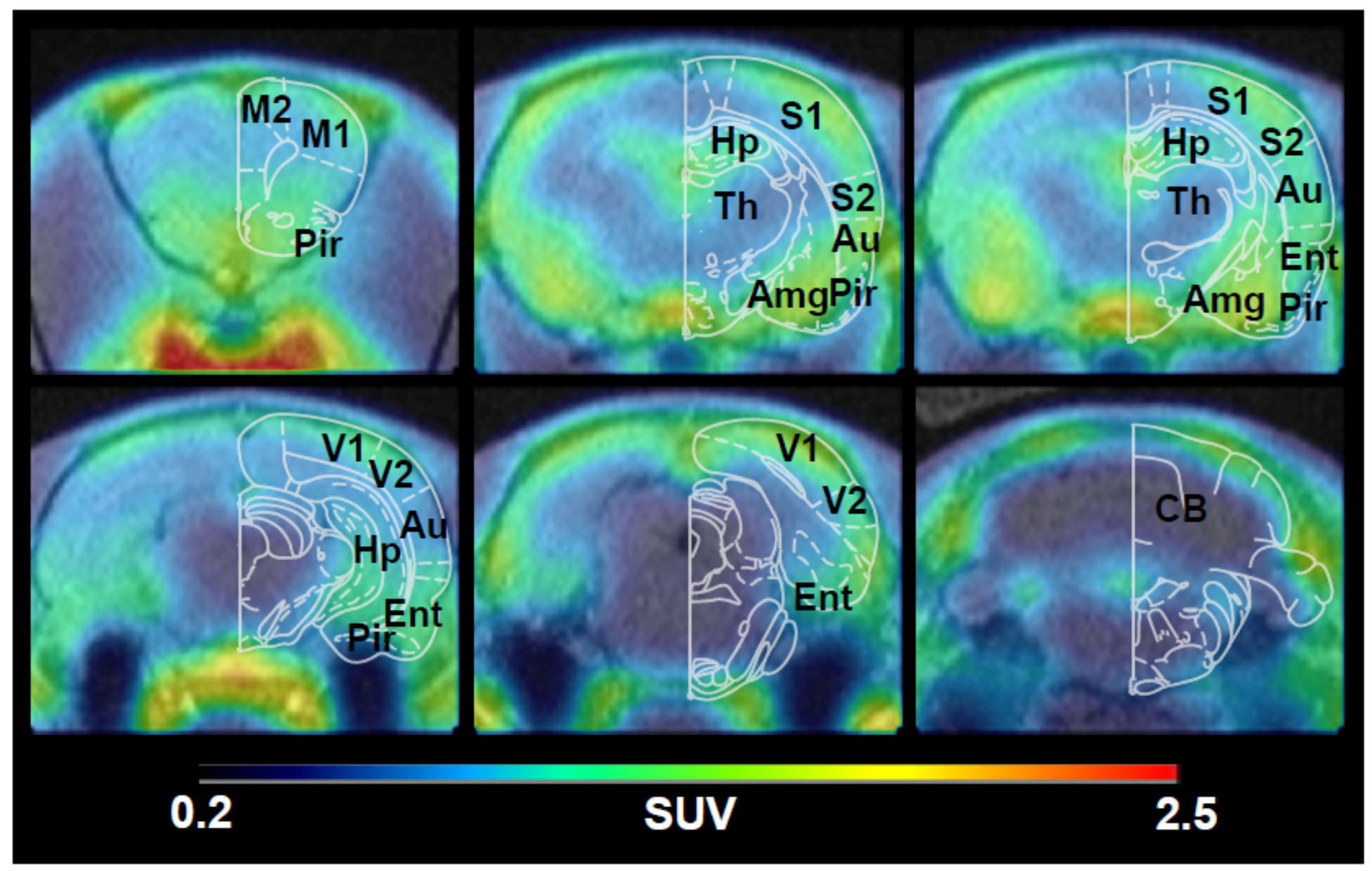

\section{Figure 1}

Representative coronal $\left[{ }^{18} \mathrm{~F}\right] \mathrm{DPA}-714$ PET images co-registered with MRI template at 3 days after induction of pharmacological status epilepticus by subcutaneous injection of KA (15 mg/kg). PET image was reconstructed with MAP algorithm and summated from 5 to $60 \mathrm{~min}$ after the bolus injection of the radioligands. PET images were expressed as standardized uptake value (SUV). Right sides of images indicate the right hemisphere. M1, primary motor cortex; M2, secondary motor cortex; Pir, piriform cortex; $\mathrm{S} 1$, primary somatosensory cortex; $\mathrm{S} 2$, secondary somatosensory cortex; Au, auditory cortex; Hp, hippocampus; Th, thalamus; Amg, amygdala; Ent, entorhinal cortex; V1, primary visual cortex; V2, secondary visual cortex; $\mathrm{CB}$, cerebellum. 

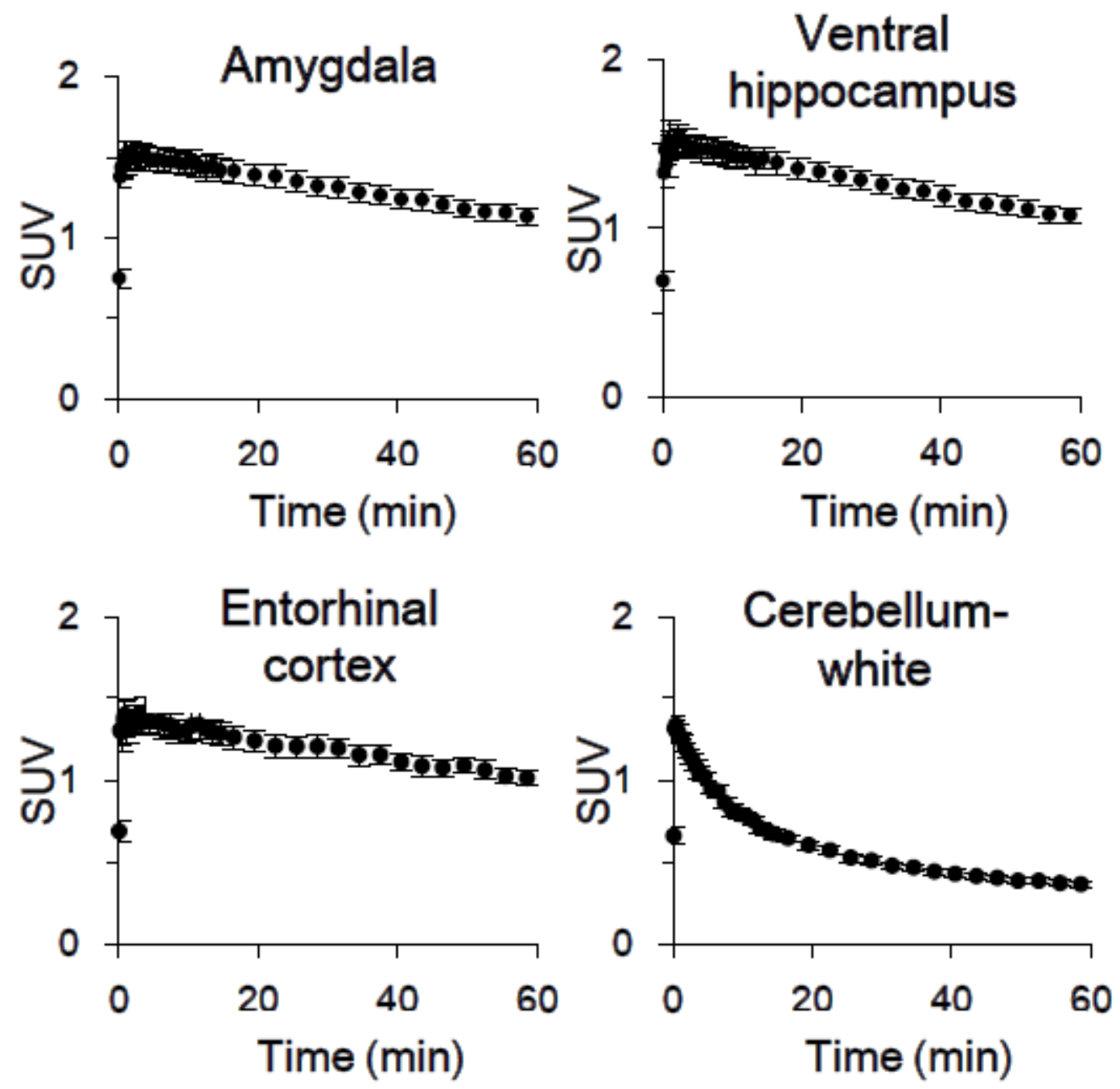

Figure 2

Time-activity curves of $\left[{ }^{18} \mathrm{~F}\right] \mathrm{DPA}-714$ in the brain regions at 3 days after status epilepticus. Decaycorrected mean values in the ROIs of Amygdala, Ventral hippocampus, Entorhinal cortex, and Cerebellumwhite were plotted over time. Each point represents mean \pm SE. ( $n=7$ animals). SUV, standardized uptake value. 


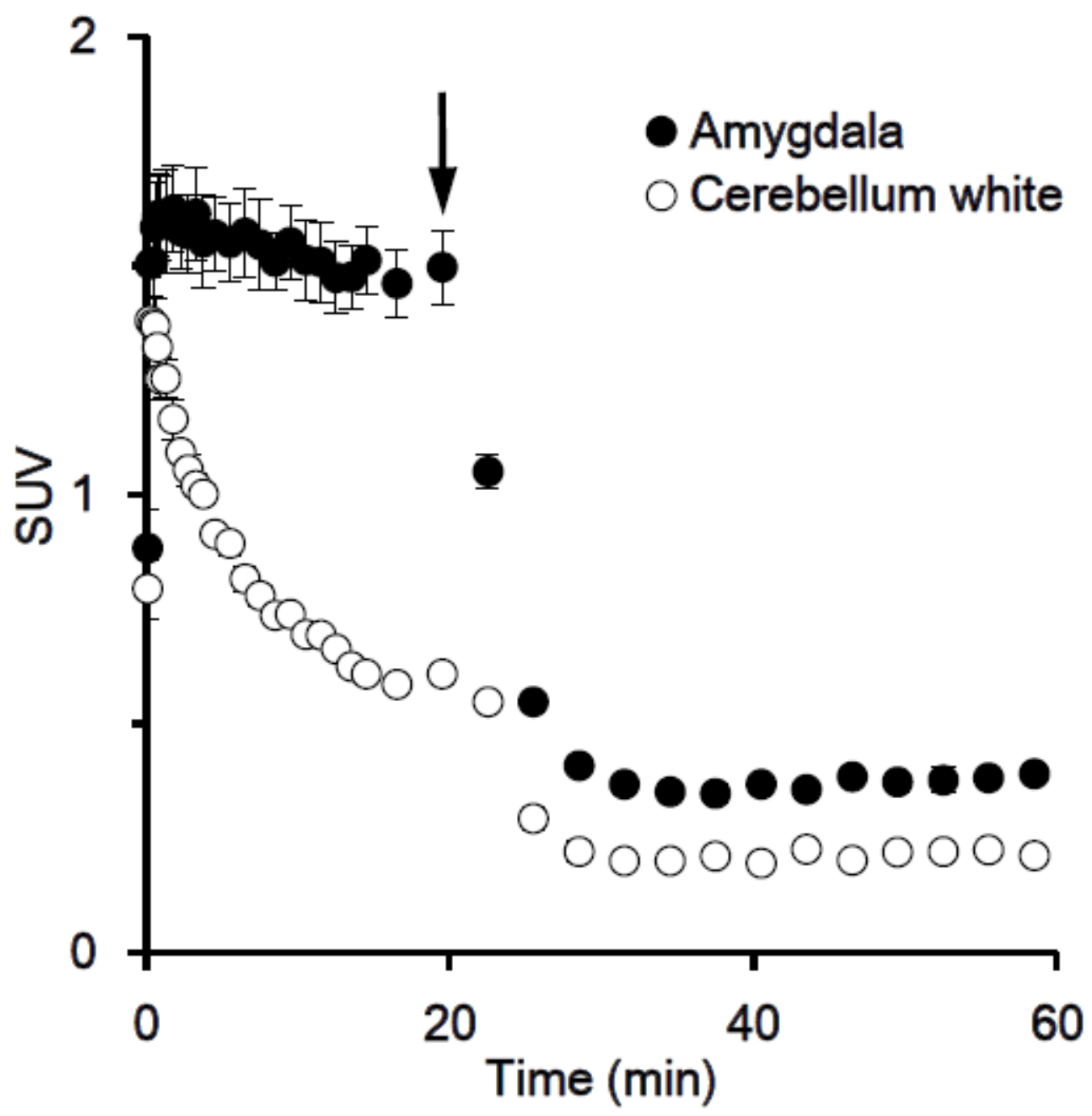

Figure 3

Time-activity curves for $\left[{ }^{18} \mathrm{~F}\right] \mathrm{DPA}-714$ displaced by excess unlabeled PK11195 in amygdala at 3 days after status epilepticus. Decay-corrected mean values in the ROls of Amygdala (closed circle) and Cerebellum-white (open circle) were plotted over time. Unlabeled PK11195 (1 mg/kg) was injected at 20 min after radio-labeled DPA-714 injection. Arrow indicates time of injection of unlabeled PK11195. Each point represents mean \pm SE. ( $n=4$ animals). SUV, standardized uptake value. 


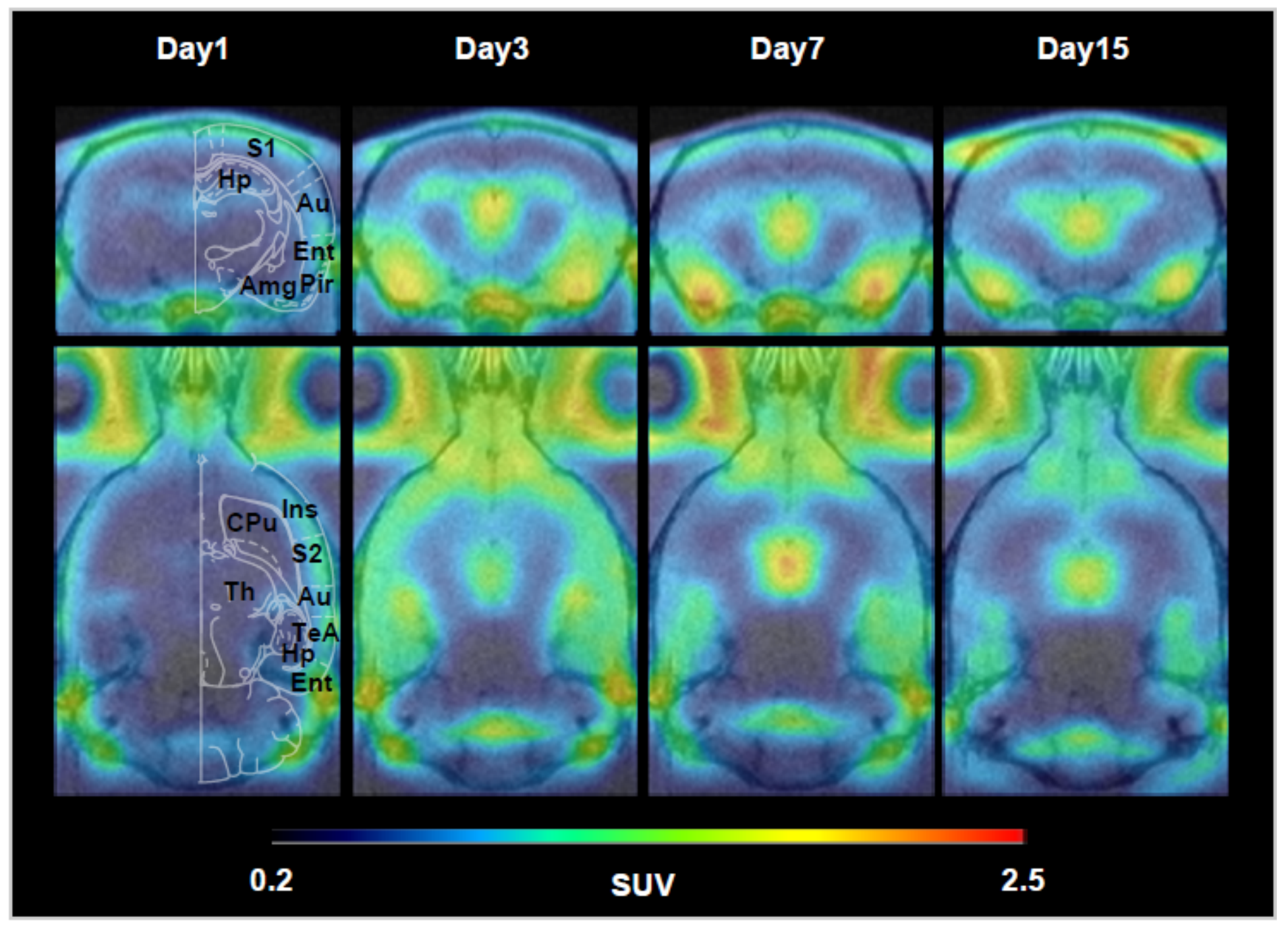

\section{Figure 4}

Representative coronal and horizontal [ $\left.{ }^{18} \mathrm{~F}\right] \mathrm{DPA}-714$ PET images co-registered with MRI template in model rats at $1,3,7$, and 15 days after induction of pharmacological status epilepticus. PET image was reconstructed with MAP algorithm and summated from 5 to $60 \mathrm{~min}$ after the bolus injection of the radioligands. PET images were expressed as standardized uptake value (SUV). Right sides of images indicate the right hemisphere. S1, primary somatosensory cortex; S2, secondary somatosensory cortex; Hp, hippocampus; Au, auditory cortex; Ent, entorhinal cortex; Amg, amygdala; Pir, piriform cortex; Ins, insular cortex; $\mathrm{CPu}$, caudate putamen; Th, thalamus; TeA, temporal association cortex. 


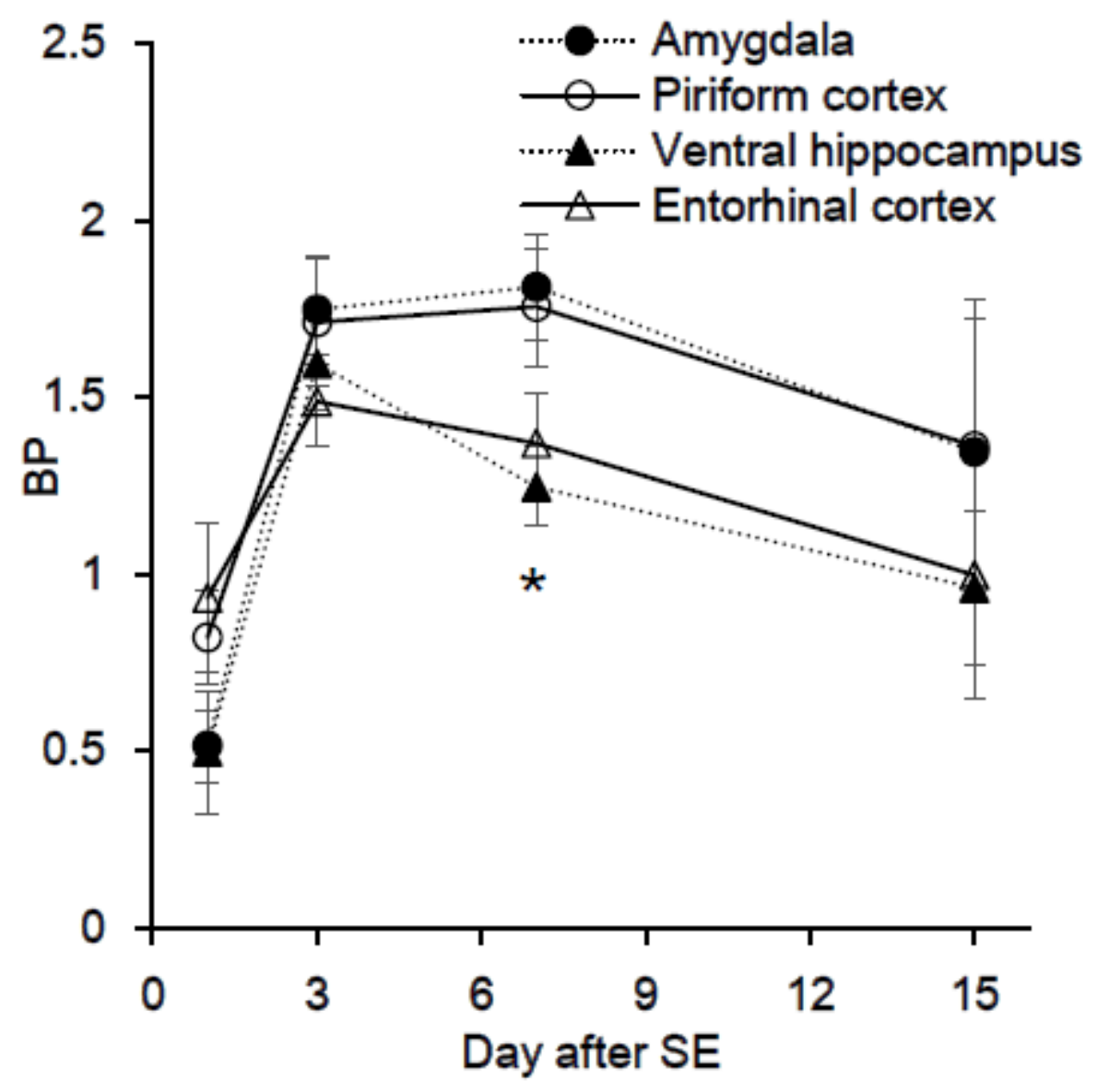

Figure 5

Temporal changes in BP values for $\left[{ }^{18} \mathrm{~F}\right] \mathrm{DPA}-714$ in the epileptogenic regions after status epilepticus.

$\mathrm{BP}$ for $\left[{ }^{18} \mathrm{~F}\right] \mathrm{DPA}-714$ in $\mathrm{KA}$-induced epileptogenic regions were estimated with simplified reference tissue model using cerebellar white matter as the reference region, at 1,3, 7, and 15 days after status epilepticus. Data are expressed as means $\pm S E(n=4$ for day $1, n=7$ for day $3, n=10$ for day 7 , and $n=4$ for day 15 , respectively, from 14 animals). ${ }^{*}, P<0.05$, amygdala vs. ventral hippocampus, two-way ANOVA followed by Bonferroni's multiple-comparison procedure. 


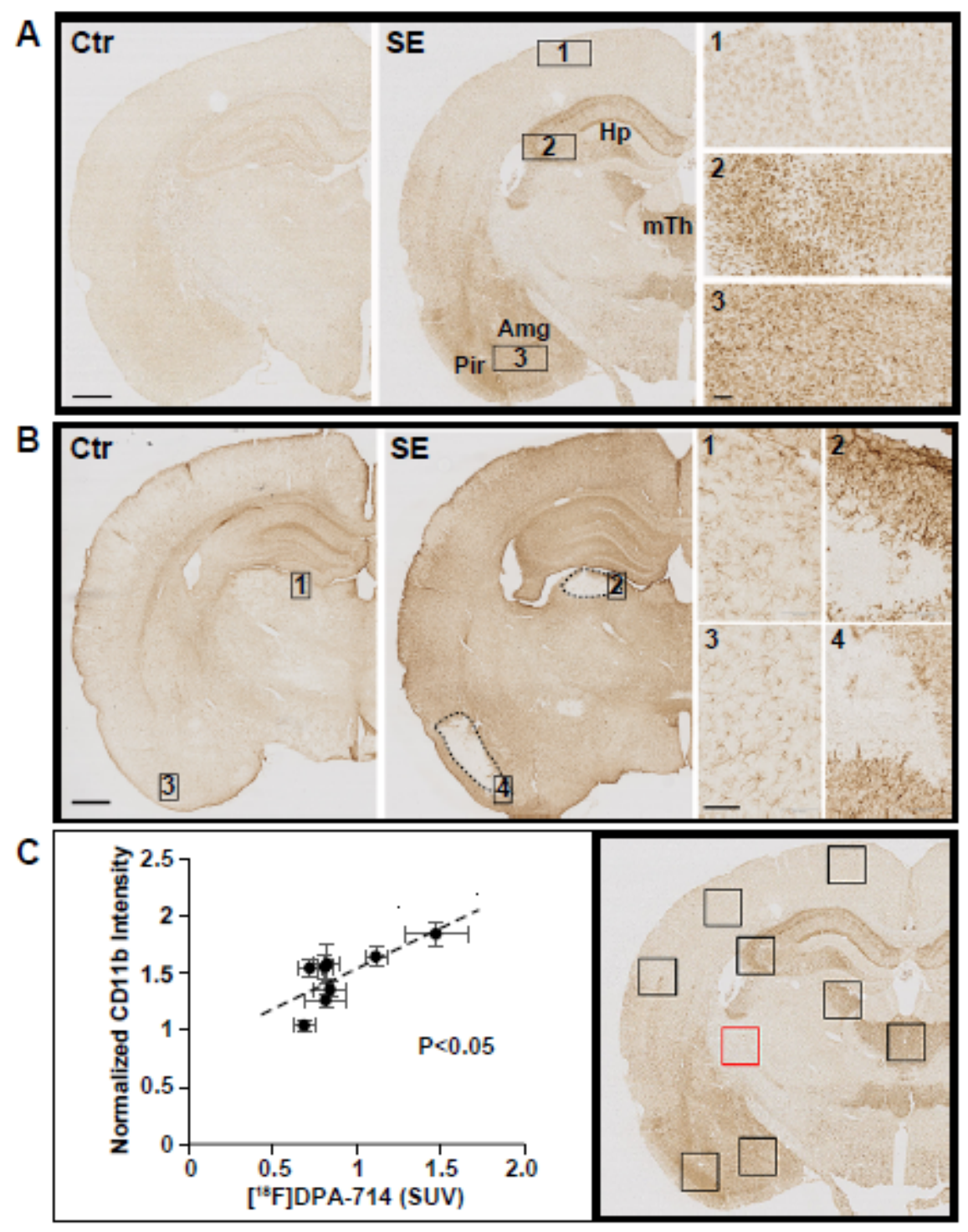

Figure 6

Immunostaining of CD11b and GFAP in control and status epilepticus model rats at 7 days after systemic administration of KA.

Photomicrographs of CD11b (A) and GFAP (B) immunoreactivity in the coronal brain sections. Dashed lines in $B$ indicate the areas show sparse GFAP-immunoreactivity. Scale bars in the left panels of $A$ and $B$ indicate $1 \mathrm{~mm}$, and those in the magnified panels of 3 in A and 3 in B indicate $100 \mu \mathrm{m}$, respectively. Ctr, control; SE, status epilepticus; Hp, hippocampus; mTh, midline thalamus; Amg, amygdala; Pir, piriform cortex. C, Correlation between the CD11b immunostaining and the corresponding [ $\left.{ }^{18} \mathrm{~F}\right] \mathrm{DPA}-714$ accumulation in the same rats. Eight black squares in the right coronal brain section indicate measurement ROIs and a red square indicates an internal reference ROI. Data are expressed as means \pm $\mathrm{SE}(\mathrm{n}=4$ animals). $P<0.05$, Pearson's correlation coefficient test.

\section{Supplementary Files}


This is a list of supplementary files associated with this preprint. Click to download.

- SeizureStage2D.mp4

- SeizureStage3G.mp4

- SeizureStage4l.mp4

- SeizureStage5K.mp4

- SeizureStage5L.mp4

- SupplementaryTable1.docx 